\title{
Full Optical Rotation Tensor at CCSD Level in the Modified Velocity Gauge
}

\author{
Kaihua Zhang, ${ }^{1}$ Ty Balduf, ${ }^{1}$ and Marco Caricato ${ }^{1, a)}$ \\ Department of Chemistry, University of Kansas, $156^{7}$ Irving Hill Road, Lawrence, Kansas 66045, \\ United States
}

This work presents the first implementation of the full optical rotation (OR) tensor at coupled cluster with single and double excitations (CCSD) level in the modified velocity gauge (MVG) formalism. The CCSDMVG OR tensor is origin independent, such that each tensor element could be in principle compared with experimental measurements on oriented systems. However, such measurements are not available for the small/medium size molecules that can be treated at CCSD level. Therefore, we compare the CCSD results with those from two density functionals, B3LYP and CAM-B3LYP, on a test set of 22 chiral molecules. The results show that the functionals consistently overestimate the CCSD results for the individual tensor components and for the trace (which is related to the isotropic OR), by 10-20\% with CAM-B3LYP and 20-30\% with B3LYP. The data show that the contribution of the electric dipole-magnetic dipole polarizability tensor to the OR tensor is on average twice as large as that of the electric dipole-electric quadrupole polarizability tensor. The difficult case of $(1 \mathrm{~S}, 4 \mathrm{~S})-(-)$-norbornenone also reveals that the evaluation of the former polarizability tensor is more sensitive than the latter. We attribute the better agreement of CAM-B3LYP with CCSD to the ability of this functional to better reproduce electron delocalization compared with B3LYP, consistently with previous reports on isotropic OR. The CCSD-MVG approach allows the computation of reference data of the full OR tensor, which may be used to test more computationally efficient approximate methods that can be employed to study realistic models of optically active materials.

Keywords: Optical Rotation, Linear Response

\section{INTRODUCTION}

Optical rotation $(\mathrm{OR})$ is one of the manifestation of the optical activity of chiral systems, where the plane of polarization of light incident onto an active sample is rotated clockwise or anti-clockwise depending on the enantiomeric excess. ${ }^{1}$ This property can be useful for the assignment of the absolute configuration of a chiral sample, which is essential for applications in biochemistry and drug design. Although most measurements and simulations of optical rotation focus on isotropic systems, e.g. in solution and in gas phase, it is also interesting to investigate oriented systems. In fact, for the latter it is possible to measure each individual element of the tensor, which can provide more information about the properties of the system. ${ }^{2,3}$ From the experimental point of view, such measurements can only be performed on crystals because of the limited intensity of the signal. These solid-state measurements are still difficult because they require a very smooth surface to distinguish the optical rotation signal (circular birefringence) from more intense signals (linear birefringence), and only a limited amount of experimental data is available. ${ }^{2,3}$

Theoretical simulations are of paramount importance for the correct assignment of the absolute configuration of chiral molecules, and many methods have been developed mostly based on density functional theory (DFT) and coupled cluster (CC) theory. ${ }^{4-19}$ However, the majority of these studies focuses on the isotropic OR

\footnotetext{
a) Electronic mail: mcaricato@ku.edu
}

because these methods were developed for molecules, not solid materials, and because of the predominance of experimental data in gas and solution phase. Thus, only few studies report the full OR tensor. ${ }^{20-24}$ The simulations of the isotropic OR are less computationally demanding than those for the full OR tensor, but they are also more prone to numerical errors that make the comparison with experimental measurements more uncertain even when highly accurate methods are employed. ${ }^{15}$ The former issue is due to the fact that forming the full OR tensor requires the evaluation of the mixed electric dipole-magnetic dipole and electric dipole-electric quadrupole polarizability tensors, while the isotropic OR only requires the former tensor. ${ }^{25}$ This is because the isotropic OR is computed as the trace of the full OR tensor, which is equal to the trace of the electric dipolemagnetic dipole polarizability tensor. In other words, the electric dipole-electric quadrupole polarizability tensor contribution to the full OR tensor is traceless and does not need to be evaluated. On the other hand, since the diagonal elements of the OR tensor are signed quantities, their sum is consistently one or two orders of magnitude smaller than their individual values. This means that a relatively small error in each element may lead to a significantly larger error in the trace and thus in the isotropic OR. Therefore, it is desirable to be able to evaluate the full OR tensor using highly accurate levels of theory despite the extra computational effort.

This work presents the implementation of the full OR tensor at $\mathrm{CC}$ level with single and double excitations (CCSD) using the modified velocity gauge (MVG) formalism. ${ }^{14,16}$ To the best of our knowledge, this is the 
first report that presents this implementation and corresponding numerical results; the only other example we are aware of where the electric dipole-electric quadrupole polarizability tensor was evaluated at CC level was in a study by Crawford and Ruud on Raman optical activity, but the tensor was evaluated in the length gauge, thus providing inherently origin dependent results. ${ }^{26}$ Using our implementation, we can compare all of the elements of the OR tensor between CC and DFT methods, and even decompose the tensor in terms of the electric dipolemagnetic dipole and electric dipole-electric quadrupole contributions. Although CCSD can currently be used only on small/medium size molecules, not amenable to experimental measurements in a fixed spatial configuration, these results can provide reference data for the assessment of more approximate but more efficient methods that can be used for comparisons with real oriented systems.

The paper is organized as follows: the theory for the evaluation of the OR tensor in the MVG formalism is described in section II, the details of the calculations are reviewed in section III, the results of the simulations are presented in section IV, a discussion of these results and concluding remarks are reported in section $\mathrm{V}$.

\section{THEORY}

The oriented Buckingham/Dunn optical activity tensor $\tilde{\mathbf{B}}$ is defined as: ${ }^{20,24,25}$

$$
\begin{gathered}
\tilde{B}_{\alpha \beta}=\frac{1}{2}\left[\operatorname{Tr}(\mathbf{B}) \delta_{\alpha \beta}-B_{\alpha \beta}\right] \\
B_{\alpha \beta}=\frac{1}{2}\left[\beta_{\alpha \beta}+\beta_{\beta \alpha}+\frac{1}{3} \sum_{\delta, \gamma}\left(\epsilon_{\alpha \gamma \delta} A_{\gamma, \delta \beta}+\epsilon_{\beta \gamma \delta} A_{\gamma, \delta \alpha}\right)\right]
\end{gathered}
$$

where $\epsilon$ is the Levi-Civita operator, $\boldsymbol{\beta}$ is the electric dipole-magnetic dipole polarizability, and $\mathbf{A}$ is the electric dipole-electric quadrupole polarizability:

$$
\begin{gathered}
\beta_{\alpha \beta}=2 \sum_{j \neq 0} \operatorname{Im} \frac{\left\langle\psi_{0}\left|\mu_{\alpha}\right| \psi_{j}\right\rangle\left\langle\psi_{j}\left|m_{\beta}\right| \psi_{0}\right\rangle}{\omega_{j}^{2}-\omega^{2}} \\
A_{\alpha, \beta \gamma}=2 \sum_{j \neq 0} \omega_{j} \operatorname{Re} \frac{\left\langle\psi_{0}\left|\mu_{\alpha}\right| \psi_{j}\right\rangle\left\langle\psi_{j}\left|\Theta_{\beta \gamma}\right| \psi_{0}\right\rangle}{\omega_{j}^{2}-\omega^{2}}
\end{gathered}
$$

where

$$
\begin{aligned}
& \boldsymbol{\mu}=-\boldsymbol{r} \\
& \boldsymbol{m}=\frac{i}{2}(\boldsymbol{r} \times \nabla) \\
& \Theta_{\beta \gamma}=-\frac{1}{2}\left(3 r_{\beta} r_{\gamma}-\delta_{\beta \gamma} r^{2}\right)
\end{aligned}
$$

are respectively the electric dipole, the magnetic dipole, and the traceless electric quadrupole operators, with the position $\boldsymbol{r}$ and gradient $\nabla$ operators implicitly summed over all the electrons of the molecule. The Greek indices denote Cartesian coordinates, $\omega$ is the frequency of the incident electromagnetic radiation while $\left|\psi_{j}\right\rangle$ and $\omega_{j}$ are the $j^{\text {th }}$ excited state wave function and excitation frequency, respectively. We use atomic units throughout the paper, except when otherwise specified. These definitions are valid for non-resonant optical activity $\left(\omega_{j} \neq \omega\right)$ calculations; resonant optical activity is discussed in greater detail elsewhere. ${ }^{1,21,25}$

For isotropic media, the observed optical rotation (OR) is commonly reported as a normalized quantity in units of $\operatorname{deg}[\mathrm{dm}(\mathrm{g} / \mathrm{mL})]^{-1}$, known as specific rotation:

$$
[\alpha]_{\omega}=\frac{-\left(72 \times 10^{6}\right) \hbar^{2} N_{A} \omega^{2}}{c^{2} m_{e}^{2} M} \operatorname{Tr}(\boldsymbol{\beta})
$$

where $\boldsymbol{\beta}$ and $\omega$ are given in atomic units, $\hbar$ is the reduced Planck's constant (J s), $N_{A}$ is Avogadro's number, $c$ is the speed of light $(\mathrm{m} / \mathrm{s}), m_{e}$ is the electron rest mass $(\mathrm{kg})$, and $M$ is the molecular mass (amu). In Eq. 6, we used the fact that the $\mathbf{A}$ contribution to $\tilde{\mathbf{B}}$ is traceless so that $\operatorname{Tr}(\tilde{\mathbf{B}})=\operatorname{Tr}(\boldsymbol{\beta})$. Therefore, in OR simulations of molecules in isotropic media, the $\mathbf{A}$ tensor is not evaluated.

On the other hand, for oriented materials, it is possible to measure the OR along an arbitrary axis, corresponding to the various elements of the $\tilde{\mathbf{B}}$ tensor. ${ }^{2,3}$ Thus, it becomes necessary to also compute the $\mathbf{A}$ tensor to make meaningful comparisons with experiment. The formal, sum-over-states definitions of $\boldsymbol{\beta}$ and $\mathbf{A}$ given in Eqs 3 and 4 are generally not used in practice, due to their slow rate of convergence. ${ }^{27}$ Instead, these polarizabilities are calculated using linear response (LR) theory, wherein the electronic density is relaxed with respect to an external perturbation. ${ }^{14,18-20,28}$ For instance, the $\boldsymbol{\beta}$ tensor is obtained as:

$$
\beta_{\alpha \beta}=\frac{1}{\omega}\left\langle\left\langle\mu_{\alpha} ; m_{\beta}\right\rangle_{\omega}\right.
$$

The elements of the $\boldsymbol{\beta}$ and $\mathbf{A}$ tensors are origin dependent, but their combination in the Buckingham/Dunn tensor in Eqs. 1-2 is origin invariant, ${ }^{25}$ as it should be for a quantity directly related to an experimental measurement. In the same way, the traces of $\tilde{\mathbf{B}}$ and $\boldsymbol{\beta}$ are also origin invariant. Unfortunately, this desirable property is strictly satisfied only when these tensors are evaluated using the exact wave function. In practical calculations, approximate methods and finite basis sets lead to values of the OR tensor that are origin dependent even when the full $\tilde{\mathbf{B}}$ tensor is evaluated. For variational methods such as Hartree-Fock (HF) and KohnSham density functional theory (DFT), origin invariance is obtained using London atomic orbitals, also known as gauge-including atomic orbitals (GIAOs) ${ }^{18,29}$ However, GIAOs are not useful for non-variational approaches like 
standard coupled cluster (CC) theory methods. For the latter, origin invariance for the isotropic OR can be obtained using a different choice of gauge for the electric dipole. Specifically, one can compute the LR function using the velocity gauge, where the electric dipole is represented by the momentum operator, $\boldsymbol{\mu}^{V}=-\boldsymbol{p}::^{14,16}$

$$
\left\langle\langle r _ { \alpha } ; m _ { \beta } \rangle _ { \omega } \rightarrow \frac { i } { \omega } \left\langle\left\langle p_{\alpha} ; m_{\beta}\right\rangle_{\omega}\right.\right.
$$

The two sides in Eq. 8 are equal only for exact methods or for approximate variational methods with an infinite basis set, otherwise, the length and velocity gauges represent distinct approaches to evaluate these tensors and provide different numerical values of the OR. A complication for the VG approach is that $\left\langle\left\langle p_{\alpha} ; m_{\beta}\right\rangle_{0} \neq 0\right.$ for finite basis sets. Coupled with the $\omega^{-2}$ factor that comes from Eqs. 7 and 8, this issue leads to an unphysical static limit for the OR with this choice of gauge. Pedersen et al. proposed to sidestep the issue by shifting the VG $\boldsymbol{\beta}$ tensor $\left(\boldsymbol{\beta}^{V}\right)$ by an amount equal to the static limit, an approach that is commonly known as modified velocity gauge (MVG) ${ }^{16}$ For variational methods, the MVG approach is more computationally demanding than the LG-GIAOs and the latter is usually preferred. However, for standard CC methods the MVG approach has represented the only viable way to obtain origin invariant isotropic OR values, until we recently introduced an origin invariant formulation of the LG method. ${ }^{30}$

In order to obtain an origin invariant $\tilde{\mathbf{B}}$ tensor at $\mathrm{CC}$ level, one can evaluate the $\mathbf{A}$ tensor using the VG electric dipole operator and the electric quadrupole operator in the form:

$$
\begin{aligned}
\Theta_{\beta \gamma}^{V} & =\frac{1}{2}\left(\Theta_{\beta \gamma}^{r p}+\Theta_{\beta \gamma}^{p r}\right) \\
\Theta_{\beta \gamma}^{r p} & =-\frac{1}{2}\left(3 r_{\beta} p_{\gamma}-\delta_{\beta \gamma} \mathbf{r} \cdot \mathbf{p}\right) \\
\Theta_{\beta \gamma}^{p r} & =-\frac{1}{2}\left(3 p_{\beta} r_{\gamma}-\delta_{\beta \gamma} \mathbf{p} \cdot \mathbf{r}\right)
\end{aligned}
$$

Similar to Eq. 7:

$$
\left\langle r_{\alpha} ; \Theta_{\beta \gamma}\right\rangle_{\omega} \rightarrow \frac{1}{\omega^{2}}\left\langle p_{\alpha} ; \Theta_{\beta \gamma}^{V}\right\rangle_{\omega}
$$

which is satisfied for exact calculations, but is only an approximation in practice. The $\omega^{-2}$ factor in Eq. 10 comes from using the momentum operator twice (once for the dipole and once for the quadrupole operators). The $\boldsymbol{\beta}^{V}$ and $\mathbf{A}^{V}$ tensors become:

$$
\begin{array}{r}
\beta_{\alpha \beta}^{V}=\frac{2}{\omega^{2}} \sum_{j \neq 0} \omega_{j} \operatorname{Re} \frac{\left\langle\psi_{0}\left|p_{\alpha}\right| \psi_{j}\right\rangle\left\langle\psi_{j}\left|m_{\beta}\right| \psi_{0}\right\rangle}{\omega_{j}^{2}-\omega^{2}} \\
A_{\alpha, \beta \gamma}^{V}=\frac{2}{\omega^{2}} \sum_{j \neq 0} \omega_{j} \operatorname{Re} \frac{\left\langle\psi_{0}\left|p_{\alpha}\right| \psi_{j}\right\rangle\left\langle\psi_{j}\left|\Theta_{\beta \gamma}^{V}\right| \psi_{0}\right\rangle}{\omega_{j}^{2}-\omega^{2}}
\end{array}
$$

but the $\tilde{\mathbf{B}}$ tensor remains as in Eqs. 1-2. As for the length gauge, the tensors in Eqs. 11-12 can be evaluated with LR methods rather than with these sum-over-states formulas. The form of the quadrupole operator in Eq. 9 ensures that the origin dependence of the $\boldsymbol{\beta}^{V}$ and $\mathbf{A}^{V}$ tensors is related to the VG electric dipole-electric dipole polarizability, ${ }^{31-33}$ such that these terms cancel out in the $\tilde{\mathbf{B}}$ tensor. The $\mathbf{A}^{V}$ tensor computed with finite basis sets also has an unphysical static limit that needs to be subtracted out as was the case for the $\boldsymbol{\beta}^{V}$ tensor. Therefore, the MVG approach needs to be employed to evaluate the $\tilde{\mathbf{B}}$ tensor correctly, but the result is a fully origin invariant Buckingham/Dunn tensor.

We implemented the MVG $\tilde{\mathbf{B}}$ tensor in GAUSSIAN $^{34}$ at CC level with single and double excitations (CCSD). To the best of our knowledge, this work presents the first implementation of the origin invariant $\tilde{\mathbf{B}}$ tensor at this level of theory, which can provide reference data for the comparison with more approximate methods. Because of the non-Hermitian nature of the CC Hamiltonian, computing the $\tilde{\mathbf{B}}$ tensor requires the evaluation of a set of perturbed $\mathrm{CC}$ amplitudes for each unique Cartesian component of the perturbation operators at three frequencies: $0,+\omega$, and $-\omega$. This results in 9 sets of amplitudes for the electric dipole, 9 for the magnetic dipole, and 15 for the electric quadrupole. However, in practice we use the non-traceless version of the quadrupole operator in Eq. 9 as available in GAUSSIAN, therefore we have six independent electric quadrupole terms (leading to 18 sets of pertubed CC amplitudes). In summary, the CCSD-MVG $\tilde{\mathbf{B}}$ tensor requires 36 sets of perturbed amplitudes (33 if we had used the traceless quadrupole form). We also implemented the DFT-MVG $\tilde{\mathbf{B}}$ tensor for the comparison discussed in section IV, which was not available in GAUSSIAN. However, the DFT implementation is considerably simpler than that for $\mathrm{CC}$, as one only needs to solve the LR equations for the VG electric dipole perturbation, and contract the corresponding perturbed density with the magnetic dipole integrals to obtain the $\boldsymbol{\beta}$ tensor and with the electric quadrupole integrals for the A tensor. Our contribution is limited to the implementation of the latter contraction, which was the only piece missing to obtain the full $\tilde{\mathbf{B}}$ tensor. Therefore, computing the DFT-MVG $\tilde{\mathbf{B}}$ tensor costs essentially the same as computing only the $\boldsymbol{\beta}^{V}$ tensor as long as the electric dipole perturbation is used in the LR equations. On the other hand, the evaluation of the $\tilde{\mathbf{B}}$ tensor at CC-MVG level is approximately twice as expensive as computing just the $\boldsymbol{\beta}^{V}$ tensor.

\section{COMPUTATIONAL DETAILS}

The molecules used in the test set are reported in Figure 1. The geometries were taken from Ref. 35 for molecules $\mathbf{1}$ and $\mathbf{2}$, from Ref. 36 for molecules $\mathbf{3 - 7}, \mathbf{1 2}, \mathbf{1 3}$, $\mathbf{1 5}$, and 18-20, from Ref. 37 for molecules 8-11, 16, and $\mathbf{1 7}$, and from Ref. 30 for molecules $\mathbf{1 4}, \mathbf{2 1}$, and $\mathbf{2 2}$. The $\tilde{\mathbf{B}}$ calculations were performed with the MVG approach 


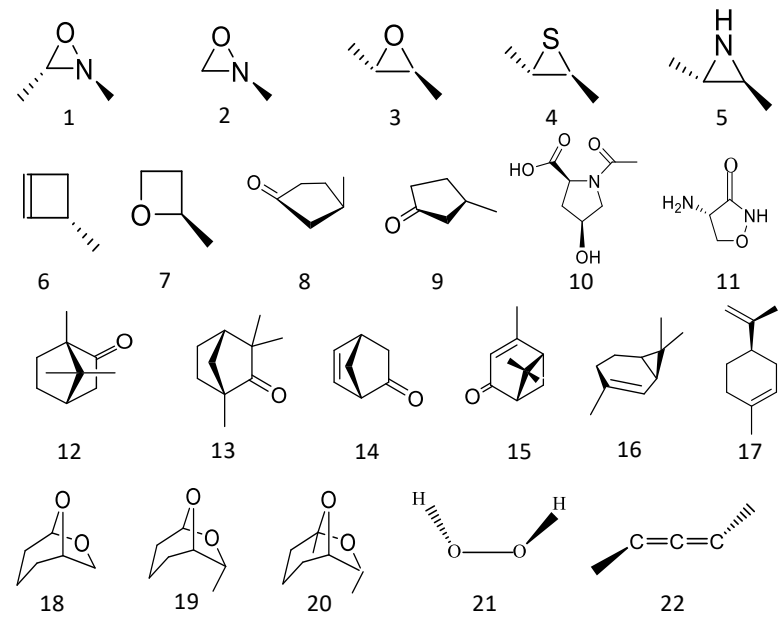

FIG. 1. Structures of the molecules in the test set.

as discussed in the previous section at the sodium $\mathrm{D}$ line: $589.3 \mathrm{~nm}$, because this is a typical wavelength used in experiment and it is far from resonance for all molecules. The OR calculations were performed with three methods: CCSD, B3LYP, ${ }^{38,39}$ and CAM-B3LYP. ${ }^{40}$ The former functional was chosen as the most popular choice in quantum chemistry, and the latter because it showed very good performance in specific rotation calculations. ${ }^{36}$ The aug-cc-pVDZ ${ }^{41}$ basis set was used for the OR calculations with all methods because it represents a reasonable costaccuracy compromise.

We assume that the CCSD results can be used as reference, and errors are defined with respect to this reference. Obviously, there is no guarantee that CCSD provides the results most in agreement with hypothetical experimental measurements. However, since CC theory provides a systematically improvable hierarchy of methods and CCSD is the best we can do at the moment, we consider it the "theoretical reference". Furthermore, in order to simplify the comparison between methods, the molecules were oriented along the principal axes of their CCSD-MVG $\tilde{\mathbf{B}}$ tensor, and the rotated structures are reported in Tables S1-S22 of the supporting information (SI). In this way, the off-diagonal elements of this tensor are zero and the corresponding values for the DFT-MVG tensor should be rather small. This allows us to concentrate our analysis on the diagonal elements of the $\tilde{\mathbf{B}}$ tensor and evaluate absolute errors for the off-diagonal elements. We also compare the $\boldsymbol{\beta}$ and $\mathbf{A}$ tensors, which are not diagonal even at CCSD level, to investigate to what extent the agreement between levels of theory is similar for these two components of the full $\tilde{\mathbf{B}}$ tensor. All of these tensors are extensive quantities, but it is better to compare intensive quantities like the specific rotation in Eq. 6. Therefore, the correlation plots in section IV use mass-scaled tensor values:

$$
T_{\alpha \beta}^{M}=\frac{T_{\alpha \beta}}{M} \times 10^{3}
$$

were $T_{\alpha \beta}$ is an element of tensor $\mathbf{T}$ in a.u., $M$ is the molar mass of the molecule in amu, and $10^{3}$ is a scaling factor used for clarity. The data for the $\boldsymbol{\beta}$ and $\mathbf{A}$ tensors are reported in terms of their linear combinations to form the $\tilde{\mathbf{B}}$ tensor, see Eqs. 1-2, rather than the actual tensor elements. The $T_{\alpha \beta}$ and $M$ values, together with the isotropic specific rotation of all molecules are reported in Tables S23-S32 of the SI. The correlation plots also include linear fits of the data with a zero-intercept constraint (an achiral molecule has zero trace with all methods). The fits are evaluated with and without molecule 14, (1S,4S)-(-)-norbornenone, because this is a notoriously difficult molecule for optical rotation calculations. ${ }^{42-44}$

\section{RESULTS}

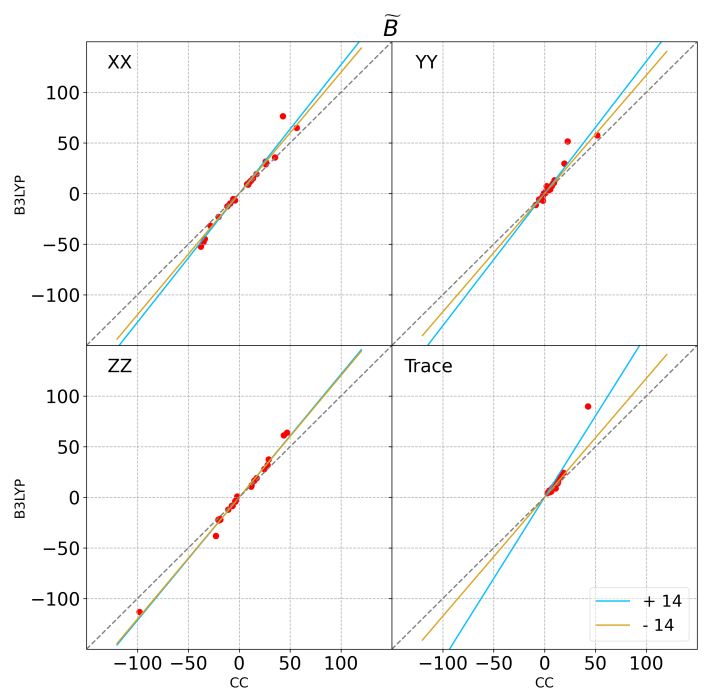

FIG. 2. B3LYP vs CCSD correlation plots for the massnormalized diagonal elements and trace (a.u. $\times 10^{3}$ ) of the $\tilde{\mathbf{B}}$ tensor, see Eq. 13. The linear fit was performed with (cyan) and without (yellow) molecule $\mathbf{1 4 .}$

Comparisons of the diagonal elements and trace of the $\tilde{\mathbf{B}}$ tensor between the density functionals and CCSD are reported in Figures 2-3. For B3LYP, the data falls fairly well on a straight line for all components and trace, but the slope is consistently larger than 1, indicating that this functional tends to overestimate the magnitude of the values compared to CCSD. The individual tensor elements ( $T_{\alpha \beta}^{M}$ in Eq. 13) are spread out over a large range of positive and negative values, but the spread of their sum (the Trace plot) is significantly smaller, which confirms the notion that in general the isotropic OR is considerably smaller than what could be measured for an oriented system. The big outlier among the data is molecule $\mathbf{1 4}$, 


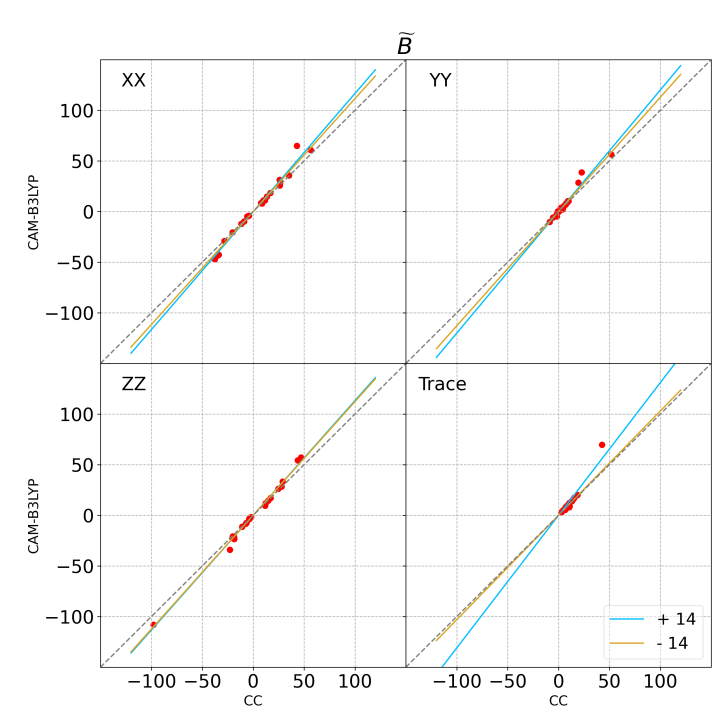

FIG. 3. CAM-B3LYP vs CCSD correlation plots for the massnormalized diagonal elements and trace $\left(\right.$ a.u. $\times 10^{3}$ ) of the $\tilde{\mathbf{B}}$ tensor, see Eq. 13. The linear fit was performed with (cyan) and without (yellow) molecule 14.

as can be seen by the difference in the fit lines in Figure 2 and a comparison of the raw data in Tables S24-S26 in the SI. However, the difference in slope between the fit lines for the individual tensor elements is considerably smaller than for the trace, which indicates that the error propagation for the isotropic OR is larger than that for the individual tensor elements. The results for CAMB3LYP are qualitatively the same as those for B3LYP, but the agreement with the CCSD results is better for the former functional. This can already be observed directly in the correlation plots in Figure 3.

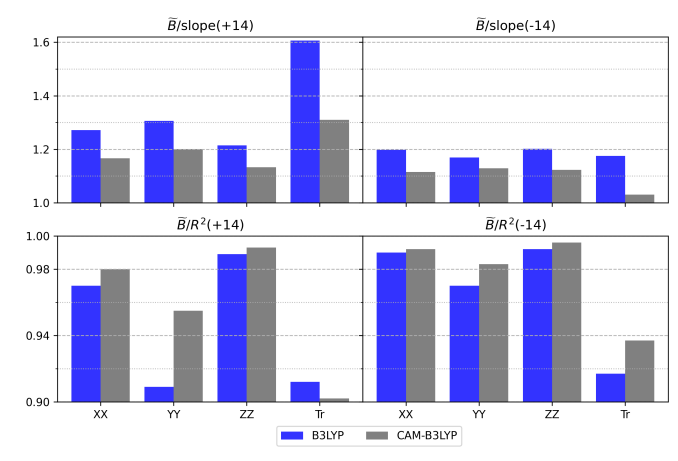

FIG. 4. Slope and $\mathrm{R}^{2}$ correlation parameters of the linear fits for the diagonal elements of the $\tilde{\mathbf{B}}$ tensor including (left-hand side plots) and excluding (right-hand side plots) molecule $\mathbf{1 4}$.

The data for the slope and $\mathrm{R}^{2}$ correlation parameter for the linear fits are reported in Figure 4. These plots indicate that B3LYP tends to overestimate CCSD by about $20-30 \%$ for the individual elements of the $\tilde{\mathbf{B}}$ tensor, but significantly more for the trace, at least when molecule $\mathbf{1 4}$ is included. This overestimation goes down to a consistent $20 \%$ without this difficult molecule. The correlation $\left(\mathrm{R}^{2}\right)$ also improves when molecule $\mathbf{1 4}$ is excluded. The $\mathrm{YY}$ and Trace $\mathrm{R}^{2}$ values are significantly smaller than the others because the range of values is smaller, so numerical noise affects the correlation of the data more significantly. The CAM-B3LYP results are considerably better than those from B3LYP across the board. The former functional overestimates CCSD by $10 \%$ for the individual tensor elements and very little for the trace when $\mathbf{1 4}$ is not included in the fitting (the better agreement for the trace is this time a sign of favorable cancellation of errors between the tensor elements). The $\mathrm{R}^{2}$ values are consistently above 0.98 for the individual tensor elements (-14) but only about 0.94 for the trace, indicating that the data correlation is worse for the $\tilde{\mathbf{B}}$ tensor trace.

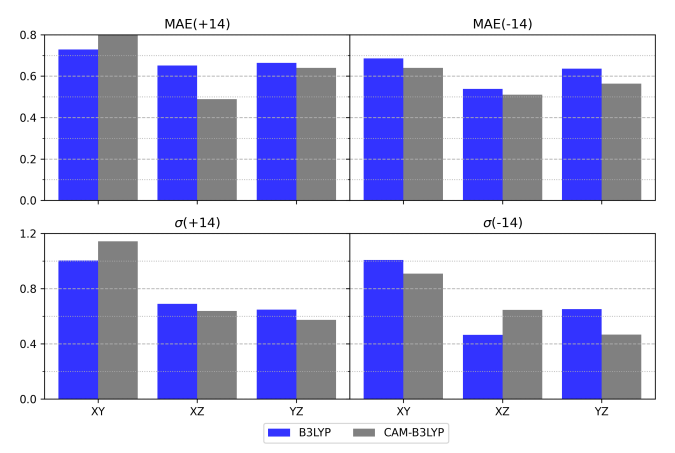

FIG. 5. Mean absolute error (MAE) and error standard deviation $(\sigma)$ for the off-diagonal elements of the $\tilde{\mathbf{B}}$ tensor including (left-hand side plots) and excluding (right-hand side plots) molecule 14 .

The off-diagonal elements of the $\tilde{\mathbf{B}}$ tensor cannot be reported in correlation plots because they are all equal to zero for CCSD given the choice of orientation of the molecules. Therefore, in Figure 5 we report the mean absolute error (MAE) and the error standard deviation $(\sigma)$, while the raw data can be found in Tables S25-S26 of the SI. The first thing to notice is that although the diagonal elements are not exactly zero, their magnitude is very small compared to that of the diagonal elements. This further confirms the overall good agreement between the CCSD and DFT results for the entire $\tilde{\mathbf{B}}$ tensor. The performance of both functionals is comparable, and the agreement with the CCSD results improves across the board if molecule $\mathbf{1 4}$ is removed from the analysis.

Although the $\tilde{\mathbf{B}}$ tensor is diagonal (or quasi-diagonal for DFT), the contributions from the $\boldsymbol{\beta}$ and $\mathbf{A}$ tensors are not. Therefore, we can compare the diagonal and offdiagonal elements in correlation plots, reported in Figures S1-S4 of the SI. Note that these plots report the contributions of the $\boldsymbol{\beta}$ and $\mathbf{A}$ tensors to the $\tilde{\mathbf{B}}$ tensor 


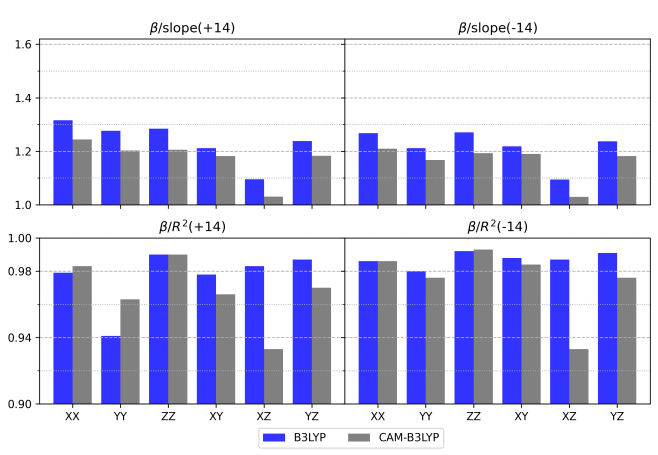

FIG. 6. Slope and $\mathrm{R}^{2}$ correlation parameters of the linear fits for the elements of the contributions of the $\boldsymbol{\beta}$ tensor to the $\tilde{\mathbf{B}}$ tensor, see Eqs. 1-2, including (left-hand side plots) and excluding (right-hand side plots) molecule $\mathbf{1 4}$.

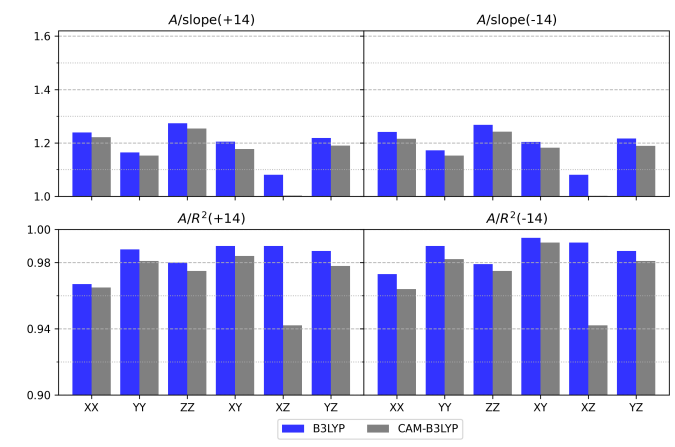

FIG. 7. Slope and $\mathrm{R}^{2}$ correlation parameters of the linear fits for the elements of the contributions of the $\mathbf{A}$ tensor to the $\tilde{\mathbf{B}}$ tensor, see Eqs. 1-2, (left-hand side plots) and excluding (right-hand side plots) molecule 14.

according to Eqs. 1-2, rather than the individual tensor elements in Eqs. 11-12. The slope and $\mathrm{R}^{2}$ values for these fits are collected in Figure 6 for the $\boldsymbol{\beta}$ tensor and in Figure 7 for the $\mathbf{A}$ tensor. The $\boldsymbol{\beta}$ tensor results indicate that B3LYP tends to overestimate CCSD by $30 \%$ for the diagonal elements and by $10-25 \%$ for the off-diagonal elements when molecule $\mathbf{1 4}$ is included. The $\mathrm{R}^{2}$ values are consistently large (around or above 0.98) except for the YY elements $\left(\mathrm{R}^{2}=0.94\right)$. When molecule $\mathbf{1 4}$ is not included in the fitting, the slope decreases by about $5 \%$ points for the diagonal elements but it stays essentially the same for the off-diagonal elements. The $\mathrm{R}^{2}$ values improve slightly for all elements, and significantly for the YY elements, which now are consistent with the other values at around 0.98. The CAM-B3LYP data show a better performance than B3LYP in terms of slope, with an overestimation of CCSD by about $20 \%$ for all elements except XZ, for which the slope is close to 1 . The $\mathrm{R}^{2}$ values are rather close to those for B3LYP, especially when molecule $\mathbf{1 4}$ is excluded from the fitting; the only exception is again the XZ elements, which are small in value and more sensitive to numerical noise.
The slope and $\mathrm{R}^{2}$ values of the linear fits for the A tensor contributions, shown in Figure 7, indicate that B3LYP does a better job with this tensor than with the $\boldsymbol{\beta}$ tensor. In fact, the slope for the diagonal elements are significantly smaller (by $5-10 \%$ points) for the XX and YY components, and mostly unchanged for the ZZ component. The $\mathrm{R}^{2}$ values are still very good, in the 0.96-0.98 range. For this tensor there is little difference between the fits that include or exclude molecule 14, indicating that the issues for this compound are due to the evaluation of the $\boldsymbol{\beta}$ tensor. The CAM-B3LYP results are very close to those for B3LYP both for the slope and $R^{2}$ values, except again for the XZ elements (for the same reason discussed in the previous paragraph). This again points to the fact that the difference between these methods in the description of the OR of molecule $\mathbf{1 4}$ is due to the $\boldsymbol{\beta}$ tensor. The fitting data for the off-diagonal elements are very close but not identical to those for the $\boldsymbol{\beta}$ tensor, compare the plots in Figures 6-7. This makes sense because the sum of the contributions of the offdiagonal elements to the full $\tilde{\mathbf{B}}$ tensor almost cancel out, leading to similar fits. These contributions would cancel out completely if the principal axes of the $\tilde{\mathbf{B}}$ tensor in the DFT calculations would perfectly coincide with those of the CCSD calculations. An interesting observation, which is hidden in the data fits of Figures 6-7 but can be noticed in the correlation plots in Figures S1-S4 of the SI, is that the range of magnitudes for the $\mathbf{A}$ tensor contributions to the diagonal elements of the $\tilde{\mathbf{B}}$ tensor is smaller by a factor of 2 compared to those of the $\boldsymbol{\beta}$ tensor. This means that about two thirds of the magnitude of the diagonal elements of the $\tilde{\mathbf{B}}$ tensor tends to come from the $\boldsymbol{\beta}$ tensor contributions and one third comes from the $\mathbf{A}$ tensor contributions. This relative partitioning is lost in isotropic OR calculations because the $\mathbf{A}$ tensor is not evaluated as its contribution to the trace of the $\tilde{\mathbf{B}}$ tensor is zero. Finally, we note that the slopes of the fitting for the diagonal elements of the $\tilde{\mathbf{B}}$ tensor are slightly smaller than those for the individual contributions of the $\boldsymbol{\beta}$ and $\mathbf{A}$ tensors, at least for the data without molecule 14, as shown by a comparison of Figures 4,6 , and 7 . The $\mathrm{R}^{2}$ parameters are in the same $0.96-0.98$ range. This indicates that there is a partial cancellation of error when the $\boldsymbol{\beta}$ and $\mathbf{A}$ tensor contributions are added up to form $\tilde{\mathbf{B}}$.

\section{DISCUSSION AND CONCLUSIONS}

In this work, we present the first implementation of the full optical rotation tensor, $\tilde{\mathbf{B}}$, at CCSD level. The MVG formalism ensures origin invariance of the tensor, such that each element is in principle comparable with experimental measurements of an oriented system. Since CCSD is applicable only on small/medium size molecules for which experimental data are not available, we focus on the comparison of the CCSD results with those obtained with two density functionals, B3LYP and CAM-B3LYP, 
on a test set of 22 chiral molecules. The results show an overall good agreement between levels of theory, especially when the difficult case of $(1 \mathrm{~S}, 4 \mathrm{~S})-(-)$-norbornenone (molecule $\mathbf{1 4}$ in the test set) is excluded from the analysis. We oriented the molecules along the principal axes of the CCSD $\tilde{\mathbf{B}}$ tensor, so that the tensor is diagonal at this level of theory and quasi-diagonal for the density functionals. The correlation plots indicate an average overestimation of the CCSD results by 20-30\% with B3LYP and $10-20 \%$ with CAM-B3LYP with correlation parameter $\mathrm{R}^{2}>0.96$, see Figure 4 . The trace of the $\tilde{\mathbf{B}}$ tensor, which is directly related to the isotropic specific rotation, is affected by the combination of the errors of the individual elements such that the slope improves (e.g., becomes closer to 1 for CAM-B3LYP) but the correlation parameter becomes worse (0.94 for CAM-B3LYP). This indicates that although the error cancellation may be overall favorable passing from the individual diagonal tensor elements to the trace, this trend is not uniform across all molecules. The overall good agreement between methods is confirmed by the small errors for the off-diagonal elements of the $\tilde{\mathbf{B}}$ tensor, see Figure 5 . These elements at DFT level are one-two orders of magnitude smaller than the diagonal elements, which indicates that the principal axes of the DFT $\tilde{\mathbf{B}}$ tensor are reasonably close to those of the CCSD tensor.

The comparison of the $\boldsymbol{\beta}$ and $\mathbf{A}$ tensor contributions to the $\mathbf{B}$ tensor shows that the $\mathbf{A}$ values tend to be smaller in magnitude than the $\boldsymbol{\beta}$ values by a factor of two. The density functionals again overestimate the CCSD results for both sets of data by about the same amount, 15-25\%, although the CAM-B3LYP results are consistently better, see Figures 6 and 7 . However, the performance on the individual $\boldsymbol{\beta}$ and $\mathbf{A}$ tensor contributions is worse than that for the full $\tilde{\mathbf{B}}$ tensor (compare these figures with Figure 4), which points to error cancellations when the tensors are combined.

When molecule $\mathbf{1 4}$ is included in the analysis, the agreement between the DFT and CCSD results worsens for the $\tilde{\mathbf{B}}$ and $\boldsymbol{\beta}$ tensor, but not for the $\mathbf{A}$ tensor. This shows that the issues with the simulations of the optical activity of this molecules are probably related to the representation of the response to the magnetic dipole perturbation. The CAM-B3LYP results are in better agreement with CCSD than those with B3LYP for the full OR tensor, which is consistent with previous reports on the specific rotation of this molecule. ${ }^{36,43,44}$ This is likely due to the fact that CCSD and CAM-B3LYP better reproduce the delocalization of the electron density across the two chromophoric groups on the molecule compared to B3LYP. This also explains the better agreement between CAM-B3LYP and CCSD across the entire test set. Although we do not explore this issue further in this report, we note that Autschbach and coworkers have shown that range-separated functionals can provide better optical activity results when the range-separation parameter is optimized to reduce the delocalization error. ${ }^{45}$

In summary, this work provides the first reference data for the full Buckingham/Dunn OR tensor for oriented systems computed at CC level. It will be interesting to investigate the basis set dependence of the full tensor calculation compared to that of the specific rotation, which we leave for a future study. Although evaluating the $\tilde{\mathbf{B}}$ tensor is almost twice as expensive as evaluating only the isotropic specific rotation at this level of theory, these data can be used as reference for more approximate but computationally efficient methods used in the study of oriented systems.

\section{SUPPLEMENTARY MATERIAL}

See the supplementary material for the geometries of the test molecules oriented along the principal axes of the CCSD-MVG $\tilde{\mathbf{B}}$ tensor (Tables S1-S22), the molecular mass and specific rotation for all molecules computed with all methods (Table S23), all elements of the $\mathbf{B}$ tensor for all molecules computed with all methods (Tables S24-S26), the contributions from the $\boldsymbol{\beta}$ tensor to the $\tilde{\mathbf{B}}$ tensor for all molecules computed with all methods (Tables S27-S29), the contributions from the $\mathbf{A}$ tensor to the $\tilde{\mathbf{B}}$ tensor for all molecules computed with all methods (Tables S30-S32), and the correlation plots for the contributions of the $\boldsymbol{\beta}$ and $\mathbf{A}$ tensors to the $\tilde{\mathbf{B}}$ tensor (Figures S1-S4).

\section{ACKNOWLEDGMENTS}

The authors gratefully acknowledge support from the National Science Foundation through Grant No. CHE-1650942.

\footnotetext{
${ }^{1}$ L. D. Barron, Molecular Light Scattering and Optical Activity, 2nd ed. (Cambridge University Press, 2004).

${ }^{2}$ W. Kaminsky, K. Claborn, and B. Kahr, Chem. Soc. Rev. 33, 514 (2004).

${ }^{3}$ J. H. Freudenthal, E. Hollis, and B. Kahr, Chirality 21, E20 (2009).

${ }^{4}$ P. L. Polavarapu, Mol. Phys. 91, 551 (1997).

${ }^{5}$ J. R. Cheeseman, M. J. Frisch, F. J. Devlin, and P. J. Stephens, J. Phys. Chem. A 104, 1039 (2000).

${ }^{6}$ S. Grimme, Chem. Phys. Lett. 339, 380 (2001).

${ }^{7}$ P. L. Polavarapu, Chirality 14, 768 (2002).

${ }^{8}$ J. Autschbach, S. Patchkovskii, T. Ziegler, S. J. a. van Gisbergen, and E. Jan Baerends, J. Chem. Phys. 117, 581 (2002).

${ }^{9}$ K. Ruud and T. Helgaker, Chem. Phys. Lett. 352, 533 (2002).

${ }^{10}$ P. J. Stephens, D. M. McCann, J. R. Cheeseman, and M. J. Frisch, Chirality 17, 52 (2005).

${ }^{11}$ S. Grimme, A. Bahlmann, and G. Haufe, Chirality 14, 793 (2002).

${ }^{12}$ K. Ruud, P. J. Stephens, F. J. Devlin, P. R. Taylor, J. R. Cheeseman, and M. J. Frisch, Chem. Phys. Lett. 373, 606 (2003).

${ }^{13}$ M. C. Tam, N. J. Russ, and T. D. Crawford, J. Chem. Phys. 121, 3550 (2004).

${ }^{14}$ T. D. Crawford, Theoretical Chemistry Accounts 115, 227 (2006).

${ }^{15}$ T. D. Crawford and P. J. Stephens, J. Phys. Chem. A. 112, 1339 (2008).

${ }^{16}$ T. B. Pedersen, H. Koch, L. Boman, and A. M. Sánchez de Merás, Chem. Phys. Lett. 393, 319 (2004).
} 
${ }^{17}$ T. D. Crawford, L. S. Owens, M. C. Tam, P. R. Schreiner, and H. Koch, J. Am. Chem. Soc. 127, 1368 (2005).

${ }^{18}$ M. Krykunov and J. Autschbach, J. Chem. Phys. 123, 114103 (2005).

${ }^{19}$ J. Autschbach, Chirality 21, E116 (2009).

${ }^{20}$ J. Autschbach, ChemPhysChem 12, 3224 (2011).

${ }^{21}$ M. Krykunov and J. Autschbach, J. Chem. Phys. 125 (2006), $10.1063 / 1.2210474$.

${ }^{22}$ V. L. Murphy and B. Kahr, J. Am. Chem. Soc. 137, 5177 (2015).

${ }^{23}$ V. L. Murphy, A. Reyes, and B. Kahr, J. Am. Chem. Soc. 138, 25 (2016).

${ }^{24}$ T. Balduf and M. Caricato, J. Phys. Chem. C 123, 4329 (2019).

${ }^{25}$ A. D. Buckingham and M. B. Dunn, Journal of the Chemical Society A: Inorganic, Physical, and Theoretical Chemistry , 1988 (1971).

${ }^{26}$ T. D. Crawford and K. Ruud, ChemPhysChem 12, 3442 (2011).

${ }^{27}$ K. B. Wiberg, Y. G. Wang, S. M. Wilson, P. H. Vaccaro, and J. R. Cheeseman, Journal of Physical Chemistry A 110, 13995 (2006).

${ }^{28}$ H. Koch and P. Jørgensen, J. Chem. Phys. 93, 3333 (1990).

${ }^{29}$ F. London, J. Phys. Radium 8, 397 (1937).

${ }^{30}$ M. Caricato, J. Chem. Phys. 153, 151101 (2020).

${ }^{31}$ P. Lazzeretti, Adv. Chem. Phys. 75, 507 (1989).

${ }^{32}$ S. Pelloni and P. Lazzeretti, J. Chem. Phys. 140, 074105 (2014).

${ }^{33}$ P. Lazzeretti, Int. J. Quantum Chem. 114, 1364 (2014).

${ }^{34}$ M. J. Frisch, G. W. Trucks, H. B. Schlegel, G. E. Scuseria, M. A. Robb, J. R. Cheeseman, G. Scalmani, V. Barone, G. A. Petersson, H. Nakatsuji, X. Li, M. Caricato, A. V. Marenich, J. Bloino, B. G. Janesko, R. Gomperts, B. Mennucci, H. P. Hratchian, J. V. Ortiz, A. F. Izmaylov, J. L. Sonnenberg, D. Williams-
Young, F. Ding, F. Lipparini, F. Egidi, J. Goings, B. Peng, A. Petrone, T. Henderson, D. Ranasinghe, V. G. Zakrzewski, J. Gao, N. Rega, G. Zheng, W. Liang, M. Hada, M. Ehara, K. Toyota, R. Fukuda, J. Hasegawa, M. Ishida, T. Nakajima, Y. Honda, O. Kitao, H. Nakai, T. Vreven, K. Throssell, J. A. Montgomery, Jr., J. E. Peralta, F. Ogliaro, M. J. Bearpark, J. J. Heyd, E. N. Brothers, K. N. Kudin, V. N. Staroverov, T. A. Keith, R. Kobayashi, J. Normand, K. Raghavachari, A. P. Rendell, J. C. Burant, S. S. Iyengar, J. Tomasi, M. Cossi, J. M. Millam, M. Klene, C. Adamo, R. Cammi, J. W. Ochterski, R. L. Martin, K. Morokuma, O. Farkas, J. B. Foresman, and D. J. Fox, "Gaussian development version revision j.11," (2020).

${ }^{35}$ P. Stephens, F. Devlin, J. Cheeseman, and M. Frisch, The Journal of Physical Chemistry A 105, 5356 (2001).

${ }^{36}$ M. Srebro, N. Govind, W. A. de Jong, and J. Autschbach, J. Phys. Chem. A 115, 10930 (2011).

${ }^{37}$ T. Aharon and M. Caricato, Chirality 32, 243 (2020).

${ }^{38}$ A. D. Becke, J. Chem. Phys. 98, 1372 (1993).

${ }^{39}$ C. Lee, W. Yang, and R. G. Parr, Phys. Rev. B 37, 785 (1988).

${ }^{40}$ T. Yanai, D. P. Tew, and N. C. Handy, Chem. Phys. Lett. 393, 51 (2004).

${ }^{41}$ T. H. Dunning Jr, J. Chem. Phys. 90, 1007 (1989).

${ }^{42}$ K. B. Wiberg, M. Caricato, Y.-g. Wang, and P. H. Vaccaro, Chirality 25, 606 (2013).

${ }^{43}$ M. Caricato, P. H. Vaccaro, T. D. Crawford, K. B. Wiberg, and P. Lahiri, J. Phys. Chem. A 118, 4863 (2014).

${ }^{44}$ P. Lahiri, K. B. Wiberg, P. H. Vaccaro, M. Caricato, and T. D. Crawford, Angew. Chem. Int. Ed. 53, 1386 (2014).

${ }^{45}$ M. Srebro and J. Autschbach, J. Chem. Theory Comput. 8, 245 (2012). 